\title{
A Novel Heparin/Protamine-Based Pro-Drug Type Delivery System for Protease Drugs
}

\author{
JUN FENG LIANG,* HUI SONG, YONG TAO LI, VICTOR C. YANG \\ College of Pharmacy, The University of Michigan, Ann Arbor, Michigan 48109-1065
}

Received 21 October 1999; accepted 20 December 1999

\begin{abstract}
Previously we proposed a heparin/protamine-based system for delivery of protease drugs such as tissue-specific plasminogen activator (tPA). To demonstrate the feasibility of this approach as well as its pro-drug and triggered release features, positively charged peptides $\left[(\mathrm{Arg})_{7} \mathrm{Cys}\right]$ were successfully linked to tissue-specific plasminogen activator (tPA) using the crosslinking agent $N$-succinimidyl-3-(2-pyridyldithio)propionate. This cation-modified tPA showed much stronger heparin affinity than the parent tPA. The complex formed by mtPA and heparin was stable in human plasma, and the activity of mtPA in such a complex was inhibited by the appended heparin. Similarly, the activity of mtPA could also be inhibited by a heparin-antifibrin IgG conjugate in which heparin was linked, via endpoint attachment, to the sugar moieties in the $\mathrm{F}_{\mathrm{c}}$ region of anti-fibrin IgG. Aside from this pro-drug feature exhibited by the binding of the macromolecule heparin to mtPA, results from chromogenic and in vitro clot lysis assay demonstrated that the heparin-induced inhibition of the mtPA activity could be easily reversed by the addition of an adequate amount of protamine. These findings suggest the applicability of the heparin/protamine delivery system to abort the potential bleeding risks associated with clinical use of tPA. In addition to the chemical conjugation method, modified tPA could also be produced by the recombinant DNA method. The expressed modified tPA (EmtPA) thus prepared retained the full catalytic activity of the parent tPA, and this activity could also be inhibited by heparin, and the heparin-induced inhibition could be reversed following the addition of protamine. (C) 2000 Wiley-Liss, Inc. and the American Pharmaceutical Association J Pharm Sci 89: $664-673,2000$
\end{abstract}

\section{INTRODUCTION}

The primary reasons for the lack of clinical application of enzymes lie in their potential immunogenicity as well as their indiscriminate actions toward both target and normal substrates. To reduce the immunogenicity and increase the life span of enzymes, much effort has been directed

Correspondence to: V. C. Yang (Email: vcyang@umich.edu)

*Visiting scholar from Department of Biological Science and Biotechnology, Life Science and Engineering School, Tsinghua University, Beijing 100084, Peoples' Republic of China.

Journal of Pharmaceutical Sciences, Vol. 89, 664-673 (2000)

(C) 2000 Wiley-Liss, Inc. and the American Pharmaceutical Association toward chemically modifying the enzyme with dextran, albumin, and polyethylene glycol, etc., or encapsulating the enzyme in human red blood cells. ${ }^{1-4}$ It has been proven that such chemical modifications and red cell encapsulation are effective in improving the pharmacokinetic profile and decreasing the immunogenicity of enzymes. The inability of an enzyme to distinguish between the target and normal substrates, however, will yield unwanted toxic effects. This still stands as the major hurdle to the use of enzymes as clinical drugs. Take thrombolytic agents as an example. These agents are plasminogen activators (PA) that are widely used in clinical treatment of established thrombus in myocardial infarction and 
pulmonary embolism. However, all these agents activate both fibrin-bound and circulating plasminogen indiscriminately. This activation of circulating plasminogen results in the systemic release of excess plasmin leading to the so-called "lytic state" manifested by marked depletion of $\alpha_{2}$-antiplasmin, fibrinogen, and other coagulation proteins. Although several approaches have been attempted to solve this problem, ${ }^{5,6}$ current thrombolytic therapy is still beset by a high incidence of bleeding complications.

Previously, we reported the development of a novel heparin/protamine-based enzyme delivery system that could be used to deliver a PA drug without this associated bleeding risk. ${ }^{7}$ As shown in Figure 1, this system comprises of a large complex made of two components: (i) a targeting component consisting of an antibody chemically linked with an anionic heparin molecule and (ii) a drug component consisting of the PA drug modified with cationic species. Because the used cations are relatively small (e.g., a small positively charged peptide), the modified PA should be able to retain a significant level of its proteolytic activity. The modified PA, however, would be deprived of such an activity once it binds to the antibody/heparin counterpart, presumably due to blocking of the PA's active sites by these appended macromolecules. The two components can be linked automatically via a tight but reversible electrostatic interaction. Therefore, similar to a "pro-drug" type approach, the modified PA would be without proteolytic activity during administration, thereby alleviating the bleeding risk introduced by systemic activation of circulating plasminogen. Following the targeting of the thrombus by the attached anti-fibrin antibody, the "active" modified-PA can be locally released at the sites of the clot by using a triggering agent such as protamine; which is the clinical heparin antagonist known to bind heparin stronger than most cationic species.

In this paper, we present a review of our recent research on the demonstration of the feasiblity of the heparin/protamine delivery system in delivery of thrombolytic agent. A poly(Arg $)_{7}$ peptide was linked, via both chemical and biological means, to a tPA molecule to produce the cationmodified drug. ${ }^{8,9}$ A novel conjugation method was established to attach heparin, via its reducing end, to the $\mathrm{F}_{\mathrm{c}}$ region of antifibrin antibody. ${ }^{9}$ This heparin anti-fibrin IgG conjugate fully retained the function of heparin for binding and of the antibody for targeting. The pro-drug and triggered

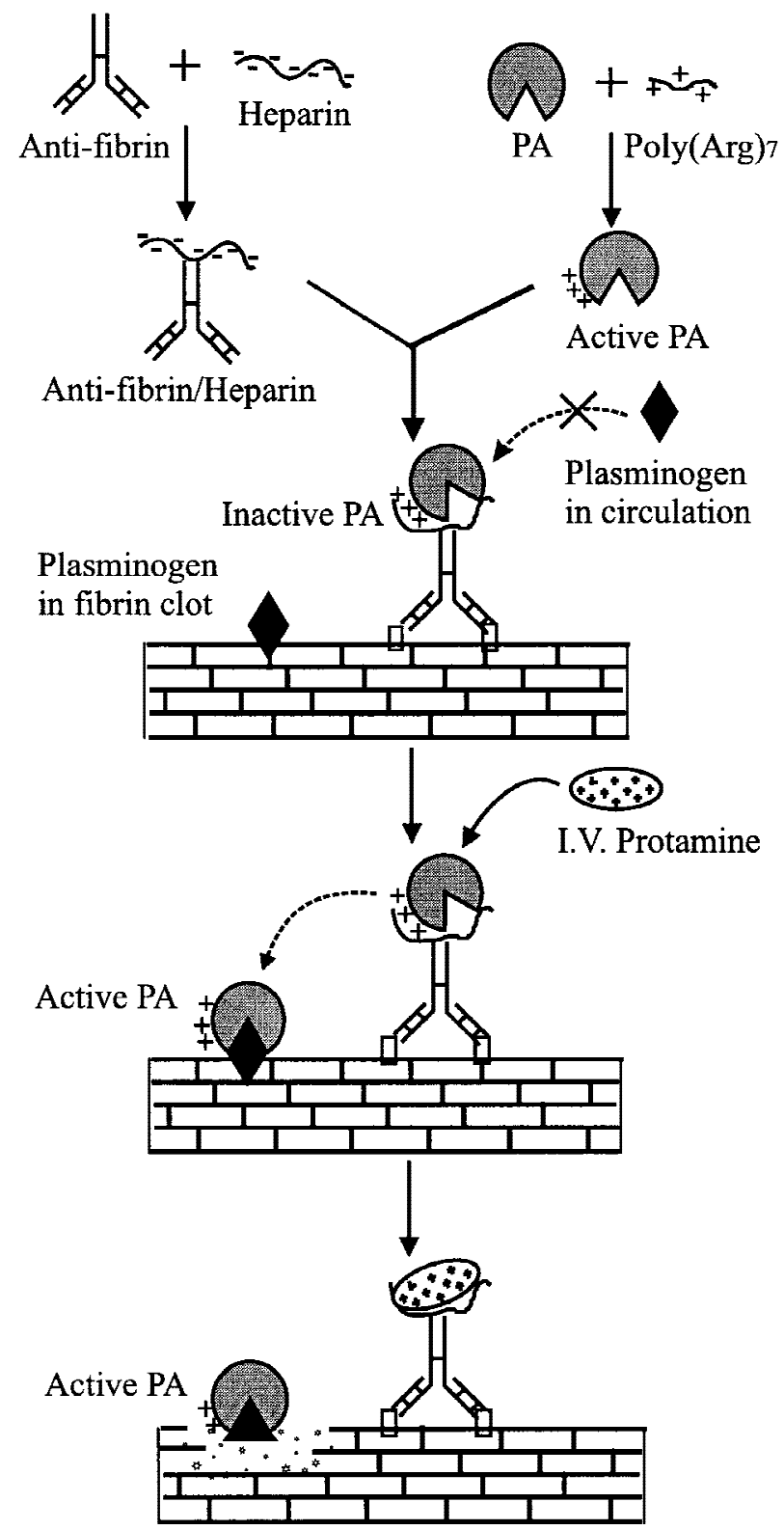

Figure 1. Schematic diagram of the proposed approach.

release features of the delivery system were successfully demonstrated in vitro using plasma samples.

\section{EXPERIMENTAL SECTION}

\section{Materials}

Recombinant tPA (Alteplase) was purchased from Genentech Inc (South San Francisco, CA). The chromogenic substrates D-Val-leu-lys-p-nitro- 
anilide (S-2251) was purchased from Pharmacia Hepar Inc. (Franklin, OH). Murine anti-fibrin and anti-tPA IgG was from American Diagnostica Inc. (Greenwich, CT). The double-stranded oligonucleotide used for expressing poly(Arg $)_{7}$ was: 5' CGCCGTCGACGGCGCAGAAGGCGCGGCGGCAGCTGCCGCGTCTTCCGCGCCGGC, which contained two NarI restriction enzyme sites at the two ends and one SalI site in between the sequence, was synthesized and purified by Integrated DNA Technologies (Coralville, IA). Plasmid PtPA-trp12 for expression of tPA in Escherichia coli and bacterial strains HB101, RB791 were obtained from ATCC (American Type Culture Collection, Rockville, MD). Other regents were from Sigma (St. Louis, MO).

\section{Preparation and Purification of the $\mathrm{tPA} /(\mathrm{Arg})_{7} \mathrm{Cys}$}

$(\mathrm{Arg})_{7}$ Cys and tPA were linked with the heterobifunctional crosslinking reagent SPDP using a two-step procedure described previously. ${ }^{10} \mathrm{~A}$ schematic description of the conjugation between $(\mathrm{Arg})_{7} \mathrm{Cys}$ and tPA was included in Figure 2A. The reaction mixture was passed through a Sephadex G-25 column to remove non-reacted reagents. Modified tPA, termed mtPA, was separated from tPA through passing a heparinSephadex column $(5 \times 0.5 \mathrm{~cm})$.

\section{Preparation of Heparin/Anti-fibrin IgG Conjugate}

Heparin $(1.0 \mathrm{~g})$ and the sugar moieties on antifibrin IgG was oxidized by sodium nitrite and $\mathrm{NaIO}_{4}$, respectively, to produce aldehyde groups. A detailed description of the chemical conjugation between heparin and anti-fibrin IgG was illustrated in Figure 2B. The heparin/anti-fibrin IgG conjugates were purified by passing through a protamine-Sepharose column and eluting with $2.0 \mathrm{M} \mathrm{NaCl}$. The anti-fibrin activity of heparin/ anti-fibrin IgG conjugate was measured by ELISA.

\section{Spectrophotometric Assay of Plasminogen Activation}

Plasminogen activation was determined by monitoring the production of plasmin using its specific substrate S-2251 according to the procedure described previously. ${ }^{11}$ The initial rate of S-2251 hydrolysis was estimated by measuring the absorbance at $405 \mathrm{~nm}$ at different time intervals in a micro-plate reader (BioRad, Hercules, CA), and was expressed as $A_{405} / \mathrm{min}^{2}$.

\section{Fibrin Clot Lysis Assay}

The activity of tPA was determined by fibrin clot lysis assay using fibrin-containing agarose plates.

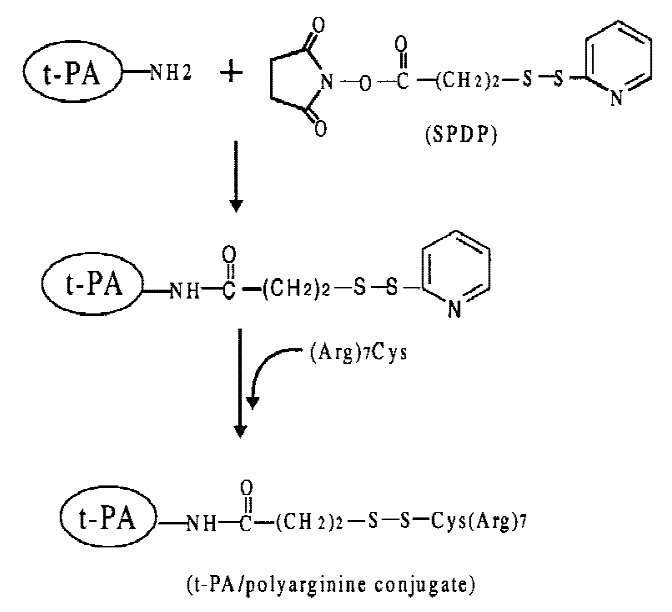

A

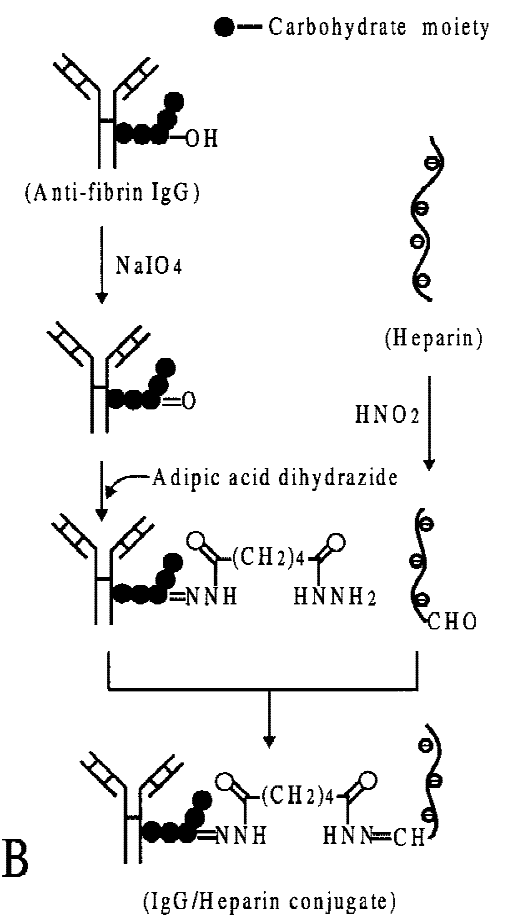

Figure 2. Schematic diagram of the synthesis of (A) modified tPA and (B) the heparin/anti-fibrin IgG conjugate. 
The plates were prepared as follows: $5.0 \mathrm{~mL}$ of low melting temperature agarose solution $(3.0 \%$, in TBS) containing $2.5 \mathrm{U}$ thrombin was mixed with $5.0 \mathrm{~mL}$ of solution containing fibrinogen (5 $\mathrm{mg} / \mathrm{mL}$ ) at $37^{\circ} \mathrm{C}$. The reaction mixtures were poured into a $90 \mathrm{~mm}$ culture dish and incubated at $37^{\circ} \mathrm{C}$ for $30 \mathrm{~min}$ until the fibrin clot became visible. To perform the assays, samples were added directly into the 3 -mm sample wells made on the solidified fibrin-agarose gel and incubated at $30^{\circ} \mathrm{C}$ for overnight. The degree of clot lysis was then estimated by analyzing the size of the fibrin lysis zone around these $3-\mathrm{mm}$ wells.

\section{Binding of tPA (or mtPA) to Heparin-Sepharose Beads}

Solutions $(0.1 \mathrm{~mL}$ ) of tPA (or mtPA) in TBS were mixed with $0.02 \mathrm{~mL}$ of heparin-Sephadex beads and incubated at room temperature for $5 \mathrm{~min}$. The heparin-Sephadex beads were then rinsed with $0.1 \mathrm{~mL}$ of plasma for $5 \mathrm{~min}$, followed by additional washing with plasma (twice) and TBS (twice). The total amount of tPA (or mtPA) activity adsorbed to the heparin beads was determined by eluting the beads with $2.0 \mathrm{M} \mathrm{NaCl}$. The percentage of inhibition of tPA (or mtPA) activity was estimated from the ratio of the tPA (or mtPA) activity adsorbed to and de-sorbed from the heparin beads.

\section{Assay of Plasminogen, $\alpha_{2}$-Antiplasmin and Fibrinogen Levels in Plasma}

Plasminogen and $\alpha_{2}$-antiplasmin levels in plasma were determined according to the method described previously. ${ }^{12}$ Fibrinogen level in plasma was estimated by determining activated partial thromboplastin time (APTT), which is inversely proportional to the fibrinogen concentration. In brief, thrombin of $500 \mathrm{U} / \mathrm{mL}(150 \mu \mathrm{L})$ was mixed with $150 \mu \mathrm{L}$ of diluted plasma and APTT was measured using a Fibrometer (BBL FibroSystem, Cockeysville, MD).

\section{Construction and Identification of the Plasmid for Expression of EmtPA in E. coli}

A schematic illustration of the procedures for construction of the plasmid for expression of EmtPA is presented in Figure 3. As shown, plasmid for expression of tPA, ptPA-trp12, was separately digested with restriction enzymes EcoRI + NarI,
$E c o \mathrm{RI}+B g l \mathrm{II}$, or NarI $+B g l \mathrm{II}$. The cleaved DNA fragments were then isolated by agarose gel electrophoresis and purified using the Nucleic Acid Rapid Isolation Kit (Sigma). A 336-base pair (bp) EcoRI-NarI fragment for encoding prior peptides, a 1,700-bp NarI-BglII fragment for expression of tPA, and a 4,200-bp vector fragment containing trp promoter and encoding gene against antibiotics were collected. These three fragments and the above double-stranded oligonucleotide (encoding the poly(Arg $)_{7}$ sequence) were linked in order using $\mathrm{T}_{4}$ ligase. After ligation, the reaction mixture was transformed into HB101 bacterial strain, and the corresponding plasmid for expression of EmtPA was screened and identified by the digestion with restriction enzymes $B g l I I$ and SalI.

\section{Expression and Purification of EmtPA}

The recombinant plasmid ( $p t P A-p o l y A r g$ ) was expressed in RB791 bacteria strain as previously described. ${ }^{13}$ After they were harvested, cells were suspended in $6 \mathrm{M}$ guanidine hydrochloride (in a volume ratio of 1:9), sonicated for $30 \mathrm{~s}$, incubated at room temperature for $30 \mathrm{~min}$, and then dialyzed overnight against $50 \mathrm{mM}$ Tris- $\mathrm{HCl}$ buffer (pH 8.0) containing $250 \mathrm{mM} \mathrm{NaCl}, 0.25 \mathrm{mM}$ EDTA, and $0.01 \%$ Tween 80 . Then the sample was loaded to $1 \mathrm{~mL}$ of HiTrap chelating column and eluted with the buffer $\left(0.02 \mathrm{M} \mathrm{Na}_{2} \mathrm{HPO}_{4}, 1 \mathrm{M}\right.$ $\mathrm{NaCl}$, and $1 \mathrm{mM}$ imidazole at $\mathrm{pH}$ 7.2) containing a linear gradient (0-0.32 M) of imidazole. The EmtPA fractions after chromatography were collected and then concentrated using an Amicon (Beverly, MA) concentrator.

\section{RESULTS AND DISCUSSION}

\section{Synthesis of the $\mathrm{tPA} /(\mathrm{Arg})_{7} \mathrm{Cys}$ and Heparin/Anti-fibrin IgG Conjugates}

It is known that the prelude of heparin-induced biological functions is its binding with antithrombin III (ATIII). This suggests that ATIII possesses a stronger affinity to heparin than all the other plasma heparin-binding proteins. ${ }^{14}$ Hence, for our approach to function properly, a pre-requisite is that the binding of the modified-PA to heparin must be stronger than that of ATIII but weaker than that of protamine. This would allow the modified-PA to remain attached to, and therefore inhibited by the appended heparin-antibody component following administration, and yet be dissociable from this component upon protamine ad- 


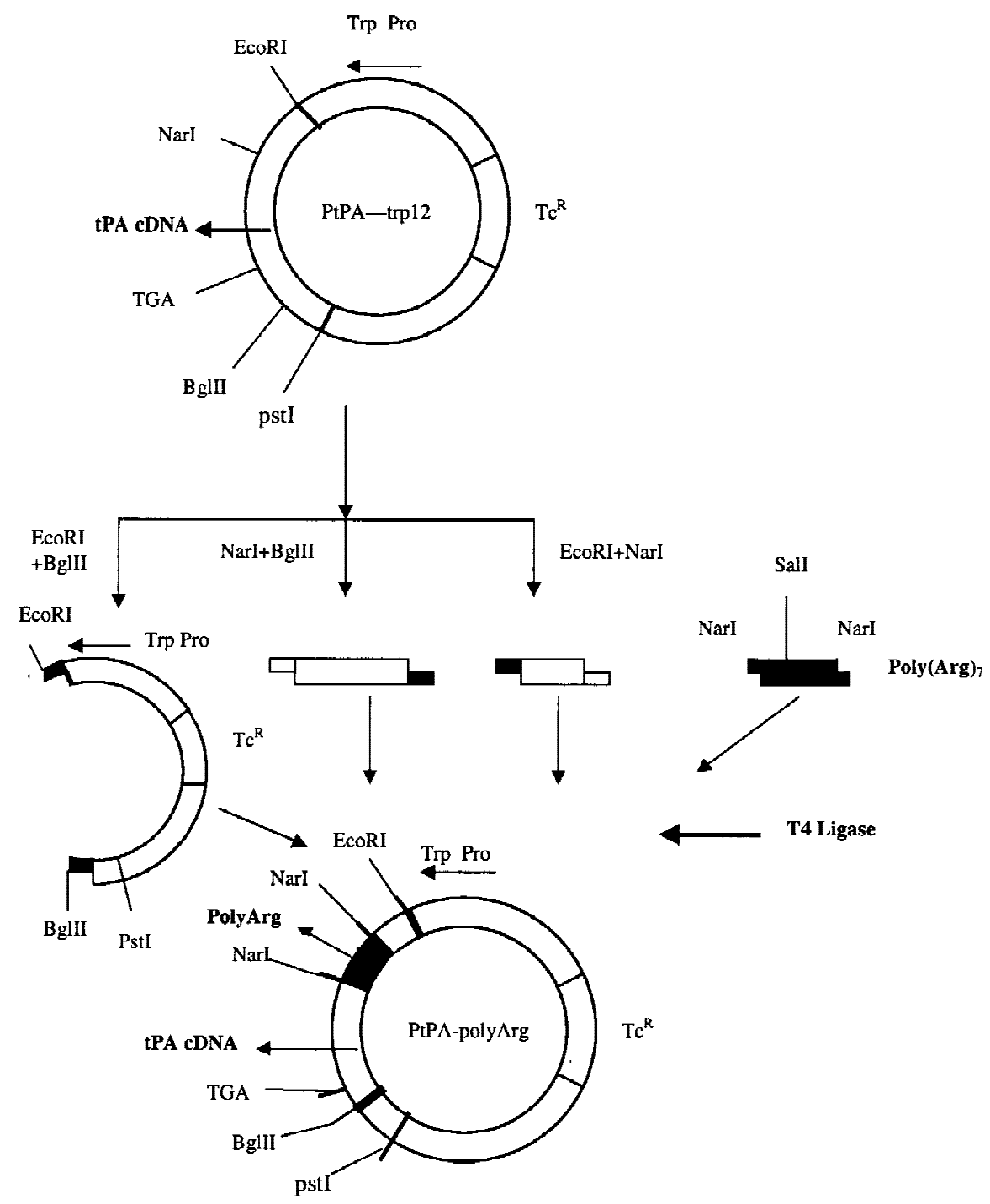

Figure 3. Schematic description of the construction of the recombinant PtPA-polyArg plasmid for expression of EmtPA.

ministration. Previous studies conducted in our laboratory indicated that a low molecular weight protamine (LMWP) fragment containing 6 or 7 arginine residues, which was prepared by digestion of native protamine with thermolysin, was able to yield a heparin-binding compount that was stronger than that of ATIII. ${ }^{15}$ On the basis of this finding, a peptide containing seven arginine residues $\left[\operatorname{poly}(\mathrm{Arg})_{7}\right]$ was attempted to produce the cation-modified tPA with the desirable heparin-binding affinity.

Figure 2A depicts the synthesis of the tPA/ (Arg) ${ }_{7}$ Cys conjugate. Approximately $40 \%$ of tPA activity was retained following the synthesis of this modified tPA (Table I). The major activity loss occurred during the purification step where the reaction mixture was purified using a heparin column. Purification of desirable modified tPA (mtPA) with a high salt elution in the removal of unmodified tPA and mtPA with weak heparinbinding affinity, resulted in a large loss in overall tPA activity recovery. This mtPA clearly contained the heparin-binding poly $(\mathrm{Arg})_{7}$ peptides, as the binding percentage of tPA in the preparation containing mtPA in TBS diluted plasma (1:1) was increased to $76 \%$ compared to that of $35 \%$ in preparation containing unmodified tPA (Table I).

Heparin was conjugated to anti-fibrin IgG via an endpoint attachment (Figure 2B). After conjugation with heparin, anti-fibrin IgG showed an increase in its molecular weight detected by SDSPAGE (Table II). The molar ratio between hepa- 
Table I. Activity Recovery and Binding to Heparin Beads Following Each Step of Preparation of the tPA/poly(Arg) ${ }_{7}$ Cys Conjugate

\begin{tabular}{lccc}
\hline \multicolumn{1}{c}{ Percentage } & $\begin{array}{c}\text { SPDP } \\
\text { Reaction }\end{array}$ & $\begin{array}{c}\text { Poly(Arg) })_{7} \text { Cys } \\
\text { Coupling }\end{array}$ & $\begin{array}{c}\text { Purification by } \\
\text { Passing Through a } \\
\text { Heparin Column }\end{array}$ \\
\hline $\begin{array}{l}\text { Recovery of total tPA activity } \\
\text { Binding to heparin beads }\end{array}$ & $\sim 100$ & $91 \pm 6$ & $36 \pm 12$ \\
\hline
\end{tabular}

${ }^{a}$ Binding percentage of tPA activity to heparin beads measured in TBS diluted human plasma (1:1) dilution.

rin and IgG in the heparin-IgG conjugate estimated by the molecular weight was consistent with that determined using azure-A and Coomassie Blue for heparin and protein quantification, respectively (Table II). Based on an average molecular mass of $15 \mathrm{kDa}$ for heparin, it was estimated that each anti-fibrin IgG was conjugated with approximately 1 or 2 heparin molecules. The anti-fibrin IgG in such heparin/anti-fibrin IgG conjugates retained nearly $80 \%$ of its original fibrin-binding ability determined by ELISA (data not shown).

\section{Characterization of the $\mathrm{tPA} /(\mathrm{Arg})_{7} \mathrm{Cys}$}

To determine if modified tPA still retained its catalytic functions, the plasmin activity produced by the mtPA-mediated plasminogen activation was measured using substrate S-2251. Compared with the specific activity of 5,800 IU/ $\mu$ g protein of the unmodified tPA, the amidolytic activity of $\mathrm{mtPA}$ was estimated to be $3,900 \mathrm{IU} / \mu \mathrm{g}$ protein. The $K_{\mathrm{m}}$ value for mtPA $(2.7 \mu \mathrm{M})$ was higher than that for tPA $(1.1 \mu \mathrm{M})$, whereas the $K_{\mathrm{m}} / k_{\text {cat }}$ data for mtPA $\left(18.2 \mu \mathrm{M}^{-1} \mathrm{~min}^{-1}\right)$ and tPA $\left(26.4 \mu \mathrm{M}^{-1}\right.$ $\mathrm{min}^{-1}$ ) were relatively comparable (Figure 4A).

It is well recognized that fibrin selectivity is the major reason for the preferred use of tPA over other clinical thrombolytic agents. Research in the past has thoroughly demonstrated that the activity of tPA was greatly enhanced in the pres-

Table II. Molecular Characterization of the Heparin-Anti-fibrin Conjugate

\begin{tabular}{lcc}
\hline & $\begin{array}{c}\text { MW by Non-reducing } \\
\text { SDS-PAGE }(\mathrm{kDa})\end{array}$ & $\begin{array}{c}\text { Heparin/IgG } \\
\text { (molar ratio) }\end{array}$ \\
\hline Heparin & 150 & $\mathrm{NA}^{\mathrm{a}}$ \\
Heparin/IgG & 175 & $1.3 \pm 0.4$ \\
\hline
\end{tabular}

${ }^{\text {a }} \mathrm{NA}$, not applicable. ence of fibrin(ogen). As shown in Figure 4B, like tPA, the mtPA-mediated plasminogen activation was also enhanced in the presence of fibrinogen, implying that mtPA still retained a similar response to fibrinogen as that of the parent tPA, and modification of tPA with the $(\mathrm{Arg})_{7} \mathrm{Cys}$ did not result in significant changes in the fibrinbinding ability of tPA.

\section{Characterization of the Pro-drug Feature of the Heparin/Protamine-Based mtPA Delivery System}

Immobilized heprin was used to mimic the macromolecular nature of the proposed heparin-IgG conjugate. To examine the pro-drug feature of the heparin/protamine-based mtPA delivery system, the amidolytic activity of tPA and mtPA bound on the heparin beads were compared. Sepharose beads containing immobilized heparin were added to the tPA or mtPA solution. To avoid any influences from loosely-bound tPA or mtPA (due to insufficient modification by the poly $(\mathrm{Arg})_{7}$ peptides), the tPA- or mtPA-bound heparin beads were rinsed three times with plasma. As shown in Table III, after extensive washing, less than $3 \%$ of tPA remained bound to the heparin beads compared to the level of $25 \%$ for mtPA. It was noteworthy that as much as $95 \%$ of the amidolytic activity of mtPA were inhibited once they were adsorbed to the heparin beads (Table III); an indication of the feasibility of the pro-drug feature of the heparin/protamine-based delivery system. In agreement with this finding, although a significant increase in plasmin and decrease in fibrinogen and plasminogen concentrations were observed when free mtPA was added into plasma, there were almost no statistically significant changes in plasma plasminogen and plasmin levels when the same amount of heparin-bound mtPA was added (Table IV). Since fibrinogen concentration was determined by APTT and the presence of heparin would cause an increase in APTT, 

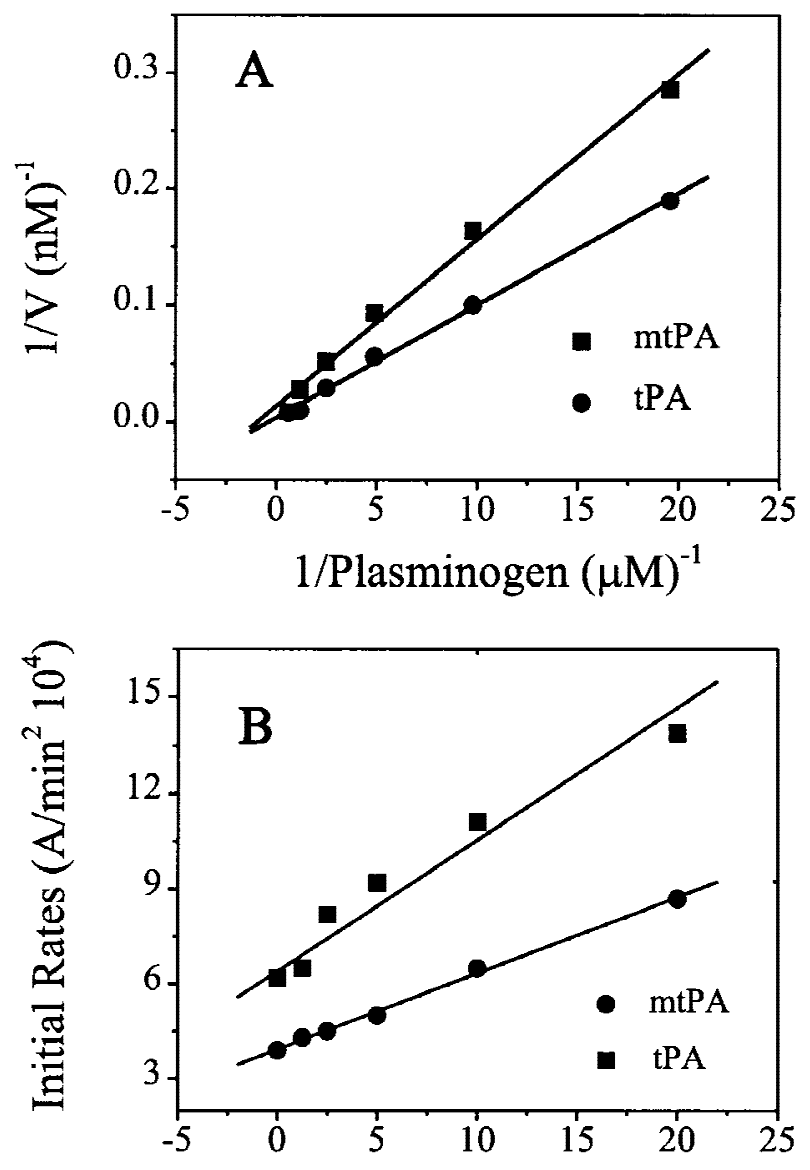

Fibrinogen Concentration (nM)

Figure 4. Characterizations of modified tPA. (A) Double-reciprocal plots for tPA and mtPA-mediated plasminogen activation. (B) Effects of fibrinogen on tPA or mtPA-mediated plasminogen activation. Experimental conditions were described in detail in the text. Rates of conversion of plasminogen to plasmin by tPA or mtPA were measured using small chromogenic substrate S-2251.

the slight decrease of fibrinogen concentration using the heparin-bound mtPA could be attributed to heparin-prolonged APTT and not necessarily to a real decrease in plasma fibrinogen concentration. The presence of nearly a similar decrease in fibrinogen concentration in plasma treated with heparin beads only (data not shown) provided support to this assumption.

To further examine the pro-drug and triggered release features of this heparin/protamine-based tPA delivery system, the in vitro fibrin clot lysis assay was performed. As shown in Figure 5, mtPA adsorbed to the heparin beads (Well 6) yielded relatively insignificant clot lysis $(<5 \%)$
Table III. Comparison Between tPA and mtPA on Binding to Heparin, Activity Inhibition by Heparin, and Activity Recovery by Protamine $(2 \mathrm{mg} / \mathrm{mL}$ ) in Plasma

\begin{tabular}{lll}
\hline \multicolumn{1}{c}{ Percentage } & tPA & mtPA \\
\hline $\begin{array}{l}\text { Binding to heparin beads } \\
\begin{array}{l}\text { Activity inhibition by binding } \\
\text { to heparin beads }\end{array}\end{array}$ & $<3$ & $25 \pm 6$ \\
$\begin{array}{l}\text { Activity recovered by reversal } \\
\text { of heparin inhibition by protamine }\end{array}$ & NA & $42 \pm 15$ \\
\hline
\end{tabular}

a NA, not applicable.

compared to that of free mtPA (Well 5). When protamine was added to the bead-bound mtPA (see Well 7), the degree of clot lysis increased, indicating that heparin-induced inhibition on the mtPA activity was reversed. These results were consistent with those seen in Table III where chromogenic assay was used. Based on these findings, it is clear that heparin can induce inhibition on the activity of mtPA via its binding to mtPA (i.e., the pro-drug feature), and this inhibition can be reversed by the addition of the triggering agent protamine (i.e., triggered release feature).

As discussed previously, the success of the discussed heparin/protamine-based delivery system relies on two key binding conditions. One is that the binding of mtPA to heparin has to be stronger than that of anti-thrombin III so that mtPA can remain attached to heparin following administration. The other requirement is that the binding of mtPA to heparin should be weaker than that of protamine so that heparin-induced inhibition on mtPA can be reversed by the addition of protamine. Previous studies conducted in our laboratory have demonstrated that the binding of poly $(\mathrm{Arg})_{7}$ to heparin is stronger than that of antithrombin III. ${ }^{15}$ Our current results obtained using the chromogenic assay (Table III) and the fi-

Table IV. Changes (\%) in Plasminogen, Plasmin, and Fibrinogen Levels in Plasma After $12 \mathrm{~h}$ of Incubation With the Same Amount $(1.3 \mu \mathrm{g} / \mathrm{mL})$ of Free mtPA or Bead-Adsorbed mtPA

\begin{tabular}{lccc}
\hline & Plasminogen & Plasmin & Fibrinogen \\
\hline $\begin{array}{l}\text { Free mtPA } \\
\text { mtPA on } \\
\text { heparin beads }\end{array}$ & $92 \pm 3$ & $120 \pm 4$ & $61 \pm 8$ \\
\hline
\end{tabular}




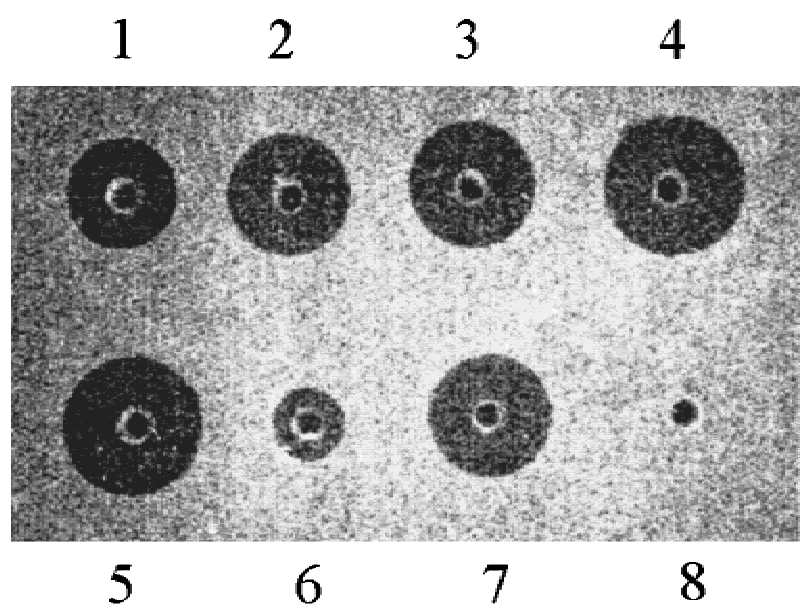

Figure 5. In vitro clot lysis assay. Experimental conditions were described in detail in the text. Wells 1-4 contain $0.025,0.05,0.1$, and $0.2 \mu \mathrm{g}$ of tPA respectively; Well 5 contains $0.2 \mu \mathrm{g}$ mtPA; Well 6 contains $0.2 \mu \mathrm{g}$ mtPA bound to heparin beads; Well 7 contains $0.2 \mu \mathrm{g}$ mtPA released from heparin beads by $50 \mu \mathrm{g}$ protamine; Well 8 contains buffer only.

brin clot lysis assay (Figure 5) have also shown that heparin-induced blockage of mtPA activity can be partially reversed by protamine. Since the binding strength of poly(Arg $)_{7}$ to heparin is comparable to that of protamine,${ }^{16}$ the concentration of protamine used for the release of mtPA from its adsorbed heparin counterpart is crucial to the reversal of heparin-induced inhibition on mtPA; as protamine at low concentrations may not be sufficient to completely dissociate mtPA from heparin binding. In addition, our recent studies using the chromogenic assay showed that at high concentrations, protamine actually inhibited tPAmediated conversion of plasminogen to plasmin (data not shown). Therefore, the lack of a $100 \%$ reversal of mtPA activity in our experiments may simply be due to the use of an inadequate amount of protamine for heparin reversal. To this end, more careful consideration of the heparin-binding affinity of the positively charged peptide (i.e., with the required heparin-binding strengths) is deemed necessary to achieve the success of utilizing the heparin/protamine-based system for tPA delivery.

As proposed in our delivery system (Figure 1), an heparin/anti-fibrin antibody conjugate was used to enhance the targeting ability of the mtPA/ heparin pro-drug conjugate. Results in Figure 6 show that like heparin beads, the heparin/antifibrin IgG conjugate was also capable of inhibiting the activity of mtPA, although this inhibition was considerably lower than that by the heparin beads $(>95 \%)$. It should be pointed out that in those experiments involving the heparin beads, only mtPA that bound strongly to the heparin beads ( $25 \%$ of total mtPA) were used. In contrast, in experiments involving the anti-fibrin/heparin conjugates, different mtPA species, with or without the sufficient heparin binding affinity, were all used in the study. Because of this lack of separation, mtPA species that were unable to bind to the heparin/anti-fibrin IgG conjugate and therefore present in the reaction mixture could exert plasminogen activation activity and, as a consequence, might contribute to the observed less inhibition by the heparin/anti-fibrin IgG conjugates.

\section{Production of Modified tPA Using Constructed mtPA-Expressing Vector}

Despite promise, however, the chemical conjugation method used previously in the preparation of mtPA was beset by a number of limitations. One is that this method was complicated and difficult to control. The produced final product was heterogeneous and contained tPA-peptide conjugates with a broad variety of heparin-binding affinities. As a consequence, the yield of the tPA-peptide conjugate with desired heparin-binding strength was relatively low; normally in the range of $30 \%$. In addition, the chemical method resulted in random incorporation of the peptides onto the tPA

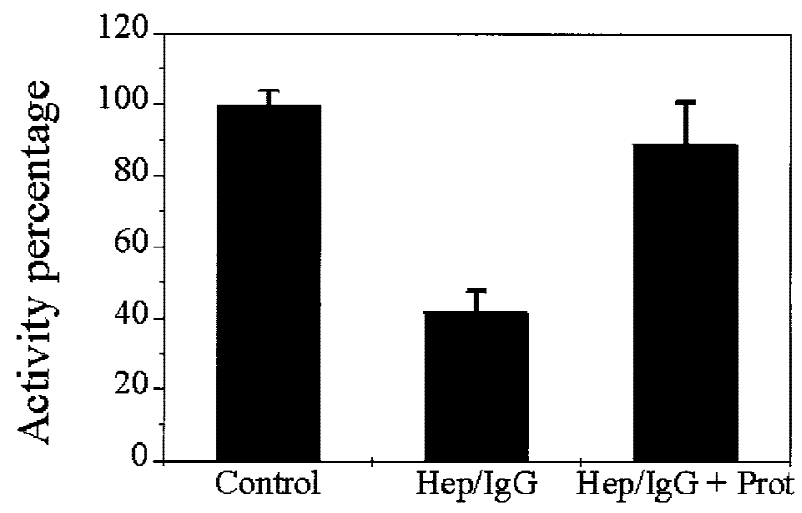

Figure 6. Inhibition of mtPA activity by the heparin/ anti-fibrin IgG conjugate and reversal of inhibition by protamine. The amidolytic activity of mtPA was measured using chromogenic substrate S-2251. Experimental conditions: $\mathrm{mtPA}, 0.5 \mu \mathrm{g} / \mathrm{mL}$; plasminogen, 0.24 $\mu \mathrm{M}$; S-2251, $1.2 \mathrm{mM}$; heparin/anti-fibrin IgG, $2 \mu \mathrm{g} / \mathrm{mL}$; protamine, $20 \mu \mathrm{g} / \mathrm{mL}$. 
protein. While incorporation of the peptide into folded areas of tPA would render it inaccessible to heparin binding, incorporation onto surface regions that were far away from the tPA active site would lead to futile heparin binding that was unable to induce any inhibitory effects. On the other hand, incorporation of the peptide by a recombinant approach would, in principle, alleviate all these limitations. To this regard, we also explored the possibility of directly producing the final tPApeptide conjugate by using recombinant DNA technology.

Figure 7 presents an identification of the constructed recombinant ptPA-polyArg plasmid. The insertion sit of poly $(\mathrm{Arg})_{7}$ is located at kringle I domain of tPA to avoid any potential influence on the thrombolytic activity and fibrin-binding ability of modified tPA. ${ }^{17}$ As shown, when digested with restriction enzymes $S a l \mathrm{I}$ and $B g l \mathrm{II}, p t P A$ trp12 was cleaved into two fragments (Lane 2) whereas ptPA-polyArg was cleaved into three fragments (Lane 4). The presence of an extra DNA band following restriction enzyme digestion

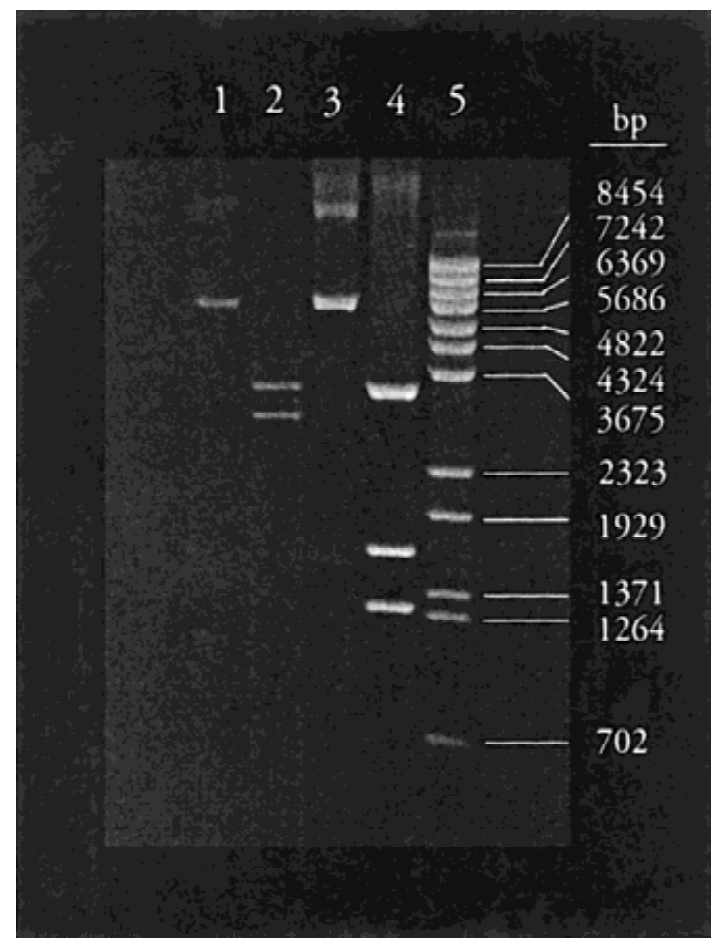

Figure 7. Identification of the recombinant $p t P A$ polyArg plasmid by restriction enzyme digestion. Lane 1, ptPA-trp12 plasmid; Lane 2, ptPA-trp12 plasmid digested with SalI and BglII; Lane 3, ptPA-polyArg plasmid; Lane 4, ptPA-polyArg plasmid digested with SalI and BglII; Lane 5, DNA markers. implicitly confirms that the additional SalI restriction enzyme site and the plasmid containing the coding sequence of poly $(\mathrm{Arg})_{7}$ has been properly inserted into $p t P A$-trp 12 .

Identification of the expressed tPA/poly $(\mathrm{Arg})_{7}$ conjugate (EtPA) was conducted by the western blot method using the anti-human tPA antibody as the probe and the anti-goat-IgG antibody conjugated with alkali phosphatase as the detecting unit. A protein band with a molecular weight slightly higher (by about 2,000 daltons) than that of the unmodified tPA (EtPA) and also responsive to the anti-tPA antibody probe was observed for the expressed protein (data not shown). Investigation of the reaction kinetics using the S-2251 chromogenic assay showed that both EtPA and EmtPA yielded almost an identical initial rate of conversion of plasminogen to plasmin under the same concentration.

Figure 8 shows that heparin enhanced the amidolytic activity of the unmodified EtPA by about $25 \%$. In a sharp contrast, heparin induced a significant inhibition of the amidolytic activity of the expressed EmtPA; on average, about 54\% inhibition of its initial activity. In addition, this heparin-induced inhibition was largely reversed when an equivalent amount of protamine (i.e., relative to heparin) was added to the heparin and EmtPA mixture (see Figure 8). These results strongly implicate that poly $(\mathrm{Arg})_{7}$ is present in the expressed protein conjugate; the inhibition is simply due to the blockage of the active site of EmtPA by the bound heparin, and the reversal of this inhibition is simply due to the dissociation of the bound heparin by protamine.

In summary, the feasibility of the heparin/

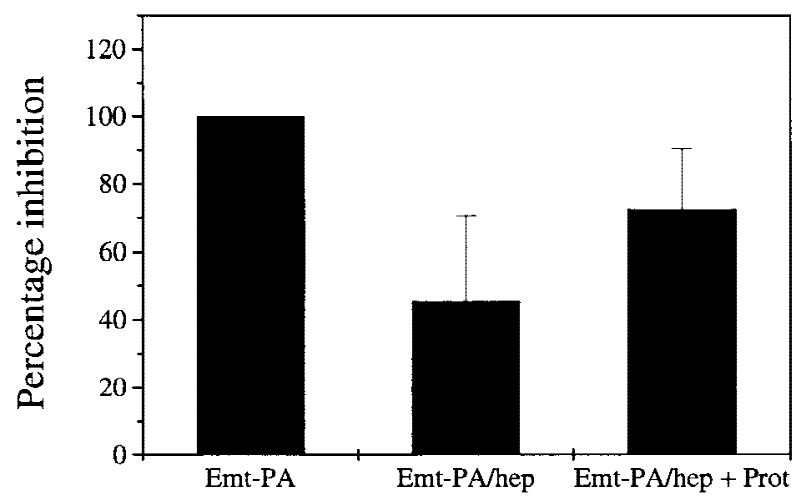

Figure 8. Inhibition of EmtPA activity by heparin and reversal of inhibition by protamine. Experimental conditions: EmtPA, $0.5 \mu \mathrm{g} / \mathrm{mL}$; plasminogen, $0.24 \mu \mathrm{M}$; S-2251, $1.2 \mathrm{mM}$. 
protamine-based approach for tPA delivery, particularly the pro-drug and triggered release features has been largely demonstrated in vitro. In addition, our results also show that it is feasible to prepare the cation-modified tPA by either chemical or the molecular biology approach. Further experiments are currently in progress in our laboratory, with the ultimate goal being aimed at demonstrating the in vivo feasibility of the proposed pro-drug delivery approach in providing targeted thrombolysis without bleeding risks.

\section{ACKNOWLEDGMENT}

This work was supported in part by National Institute of Health Grant HL55461.

\section{REFERENCES}

1. Wileman TE, Foster RL, Elliott PN. 1986. Soluble asparaginase-dextran conjugates show increased circulatory persistence and lowered antigen reactivity. Pharm Pharmacol 38:264-271.

2. Breton J, Pezzi N, Molinari A, Bonomin L, Lansen J, Gonzalez de Buitrago, Prieto I. 1995. Prolonged half-life in the circulation of a chemical conjugate between a prourokinase derivative and human serum albumin. Eur J Biochem 231:563-569.

3. Teske E, Rutteman GR, Van Heerde P, Misdorp W. 1990. Polyethylene glycol-L-asparaginase versus native L-asparaginase in canine non-Hodgkin's lymphoma. Eur J Cancer 26:891-895.

4. Kravtzoff R, Debois I, Lamagnere JP, Muh JP, Valat C, Chassaigne M, Colombat P, Ropars C. 1996. Improved pharmacodynamics of L-asparaginase-loaded in human red blood cells. Eur J Pharmacol 49:465-470.

5. Ikenaka Y, Yajima K, Yahara H, Maruyama H, Matsumoto K, Okada K, Ueshima S, Matsuo O. 1992. Characterization of human tissue-typeplasminogen activator variants with amino acid mutations in the kringle 1 domain. Blood Coagulation Fibrinolysis 3:381-387.
6. Declerck PJ, Van Keer L, Verstreken M, Collen D. 1992. An enzyme-linked immunosorbent assay for urokinase-type plasminogen activator (u-PA) and mutants and chimeras containing the serine protease domain of u-PA. Thromb Haemost 67:95-100.

7. Byun Y, Yang VC. 1998. Delivery system for targeted thrombolysis without the risk of hemorrhage. ASAIO J 44:M638-641.

8. Song H, Liang JF, Yang VC. 1999. A recombinant tissue plasminogen activator, (t-PA)-POLY(ARG) chimera, for targeted thrombolysis without the bleeding risk. ASAIO J, in press.

9. Liang JF, Li YT, Connell M, Yang VC. 1999. Synthesis and characterization of positive charged tPA as a prodrug using heparin/protamine drug delivery system. Pharm Sci, submitted.

10. Turpie AGG. In: Sobel BE, Collen D, Grossbard EB, editors. 1987. Tissue plasminogen activator in thrombolytic therapy. New York: Marcel Dekker. p 131-146

11. Bernstein H, Yang VC, Langer R. 1987. An investigation of heparinase immobilization. Appl Biochem Biotech 16:129-143.

12. Yun JH, Ma SC, Fu B, Yang VC, Meyerhoff ME. 1993. Direct potentiometric membrane-electrode measurements of heparin-binding to macromolecules. Electroanalysis 5:719-724.

13. Harris TJ, Patel T, Marston FAO, Little S, Emtage JR. 1986. Cloning of cDNA coding for human tissue-type plasminogen activator and its expression in Escherichia coli. Mol Biol Med 3:279-292.

14. Jaques LB. 1980. Heparin-anionic poly-electrolyte drugs. Pharmacol Rev 31:99-166.

15. Byun Y, Singh VK, Yang VC. 1999. Low molecular weight protamine: a potential nontoxic heparin antagonist. Thromb Res 94:53-61.

16. Ramamurthy N, Baliga N, Wakefield TW, Andrews PC, Yang VC, Meyerhoff ME. 1999. Determination of low-molecular-weight heparins and their binding to protamine and a protamine analog using polyion-sensitive membrane electrodes. Anal Biochem 266:116-124.

17. Lijnen HR, Collen D. 1991. Strategies for the improvement of thrombolytic agents. Thromb Haemost 66:88-110. 